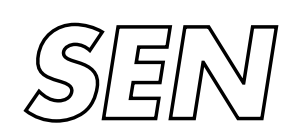

Software Engineering

$\int$ SEN Strongly typed heterogeneous collections

O. Kiselyov, R. Lämmel, K. Schupke

Report SEN-E0420 November 2004 
CWI is the National Research Institute for Mathematics and Computer Science. It is sponsored by the Netherlands Organization for Scientific Research (NWO).

$\mathrm{CWI}$ is a founding member of ERCIM, the European Research Consortium for Informatics and Mathematics.

CWI's research has a theme-oriented structure and is grouped into four clusters. Listed below are the names of the clusters and in parentheses their acronyms.

Probability, Networks and Algorithms (PNA)

\section{Software Engineering (SEN)}

Modelling, Analysis and Simulation (MAS)

Information Systems (INS)

Copyright (C) 2004, Stichting Centrum voor Wiskunde en Informatica

P.O. Box 94079, 1090 GB Amsterdam (NL)

Kruislaan 413, 1098 SJ Amsterdam (NL)

Telephone +31205929333

Telefax +31205924199 


\title{
Strongly Typed Heterogeneous Collections
}

\author{
ABSTRACT \\ A heterogeneous collection is a datatype that is capable of storing data of different types, while \\ providing operations for look-up, update, iteration, and others. There are various kinds of \\ heterogeneous collections, differing in representation, invariants, and access operations. We \\ describe HList --- a Haskell library for strongly typed heterogeneous collections including \\ extensible records. We illustrate HList's benefits in the context of type-safe database access in \\ Haskell. The HList library relies on common extensions of Haskell 98. Our exploration raises \\ interesting issues regarding Haskell's type system, in particular, avoidance of overlapping \\ instances, and reification of type equality and type unification.
}

1998 ACM Computing Classification System: E.2; D.2.13; D.3.1

Keywords and Phrases: Collections; Extensible records; Type-safe database access; Dependently typed programming; Type-indexed rows; Type equality; Type improvement; Haskell 



\title{
Strongly Typed Heterogeneous Collections
}

\author{
August 26, $2004^{*}$
}

\author{
Oleg Kiselyov \\ FNMOC, Monterey, CA
}

\author{
Ralf Lämmel \\ VU \& CWI, Amsterdam
}

\author{
Keean Schupke \\ Imperial College, London
}

\begin{abstract}
A heterogeneous collection is a datatype that is capable of storing data of different types, while providing operations for look-up, update, iteration, and others. There are various kinds of heterogeneous collections, differing in representation, invariants, and access operations. We describe HLIST - a Haskell library for strongly typed heterogeneous collections including extensible records. We illustrate HLIST's benefits in the context of type-safe database access in Haskell. The HLIST library relies on common extensions of Haskell 98. Our exploration raises interesting issues regarding Haskell's type system, in particular, avoidance of overlapping instances, and reification of type equality and type unification.
\end{abstract}

Categories and Subject Descriptors: E. 2 [Data Storage Representations]; D.2.13 [Software Engineering]: Reusable Software; D.3.1 [Programming Languages]: Formal Definitions and Theory

General Terms: Design, Languages.

Keywords: Collections, Extensible records, Type-safe database access, Dependently typed programming, Type-indexed rows, Type equality, Type improvement, Haskell.

\section{Introduction}

Programmers in typed functional languages are used to homogeneous collections, where values of the same type are stored in lists, sets, and others. There exist collection libraries, e.g., Edison for Haskell [26]. Homogeneous collections rely on parametric polymorphism. C++ programmers are also used to homogeneous collections such as those in the Standard Template Library, likewise for Ada and Eiffel. Java programmers are about to receive support for parametric polymorphism, finally. This may end the use of weakly typed collections ("Everything is of type Object!"), which require run-time type casts with the potential of unappreciated exceptions.

Unfortunately, the notion of typeful homogeneous collections fails to work for all the scenarios that require storing values of different types. Here is an open-ended list of typical examples that call for heterogeneous collections:

- A symbol table that is supposed to store entries of different types is heterogeneous. It is a finite map, where the result type depends on the argument value.

- An XML element is heterogeneously typed. In fact, XML elements are nested collections that are constrained by regular expressions and the 1-ambiguity property.

${ }^{*}$ A shorter version of this paper appeared in the proceedings of the ACM SIGPLAN Haskell Workshop 2004, September 22, 2004, Snowbird, Utah, USA, Published by ACM Press. This longer version provides several appendices and some extra paragraphs.
- Each row returned by an SQL query is a heterogeneous map from column names to cells. The result of a query is a homogeneous stream of heterogeneous rows.

- Adding an advanced object system to a functional language requires heterogeneous collections of a kind that combine extensible records with subtyping and an enumeration interface. Weakly typed encodings are feasible for all the listed scenarios. For instance, a heterogeneously typed symbol table can be encoded using a suitably universal type, or dynamic typing, or type-safe cast.

The present paper introduces a strong typing discipline for heterogeneous collections. We deliver a dedicated Haskell library HLIST, which covers collection types such as lists, arrays, extensible records, type-indexed products and co-products. To this end, we advance techniques for dependently typed programming in Haskell [12, 21], and we rely on Haskell 98 with common extensions for multi-parameter classes and functional dependencies, as available in the GHC and Hugs implementations. (We manage to avoid overlapping instances - in the end!) Our development does not introduce yet another language extension, which is an improvement over earlier proposals for extensible records and other collection types $[10,31,23,29]$. We explore some murky waters of Haskell's type system, such as the reification of type equality and type unification. While we have found portable, sound and practical ways around, more research is needed to deliver foundational clarifications that enable fundamental solutions. We identify the issues that need to be resolved.

The paper is structured as follows. In Sec. 2, we review weakly typed techniques for dealing with heterogeneous collections. In Sec. 3, we introduce typeful heterogeneous lists, which provide the basis for the HLIST library. We then work out different kinds of access operations and collection types:

- Sec. 4 - numeral-based access operations,

- Sec. 5 - labelled collections (or records),

- Sec. 6 - type-based access operations,

- Sec. 7 - type-indexed products.

In Sec. 8, we demonstrate the merits of heterogeneous collections in the context of type-safe database access in Haskell. In Sec. 9, we review our take on Haskell's type system. In Sec. 10, we discuss related work, and we conclude in Sec. 11. There are several appendices with various details. The source code from the paper can be retrieved from [1]. 


\section{Not so strongly typed collections}

We use database programming for the motivation of the HLIST library in this paper. We want to get to a point where SQL queries can be rephrased in Haskell in a typed and structured manner. As a simple example, let us attempt to encapsulate a simple SQL query in a Haskell function. The query should retrieve all animals (their keys and names) of a given breed from the 'foot-n-mouth' database. A query for sheep (rather than cows) looks as follows:

SELECT key, name FROM Animal WHERE breed = ' sheep';

\section{Cheap strings}

The following Haskell code encodes the parameterised query:

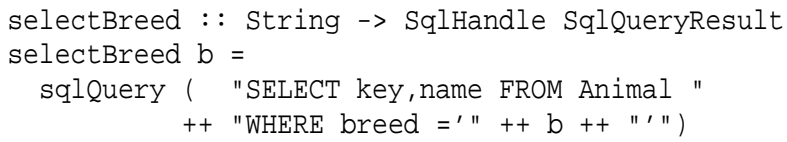

Here we use a low-level ODBC binding for database access. The query is wrapped in an SqlHandle type, which encapsulates an IO action for an ODBC connection. The query function is parameterised in a String for the breed parameter. The type of query results is defined as follows:

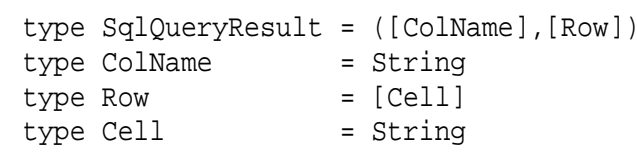

That is, the result of a query consists of a list of column names and a list of rows, where a row in turn is a list of cells. Both column names and cells are plain strings. This is painful code in the eye of most programmers, but it is often a cheap way to make things work. Prominent database access techniques for all kinds of programming languages are string-based just like that.

\section{Hand-made universes}

If we wanted to maintain at least the primitive datatypes of cells, then we could replace the use of the string type with a universe of cell types (or a tagged union):

$$
\begin{aligned}
& \text { data Cell }=\text { Intobject Int } \\
& \mid \begin{array}{l}
\text { Floatobject Float } \\
\text { Stringobject String } \\
\ldots-- \text { and perhaps a few more cases }
\end{array}
\end{aligned}
$$

A row is still a list of such cells, but it is effectively a heterogeneous list. Instead of Int and Intobject we can use types and tags that are more descriptive of the columns, as below. Clearly, such an application-specific universe is subject to change whenever the data dictionary changes.

These are the types for the columns in the 'foot-n-mouth' database:

$$
\begin{array}{llll}
\text { newtype Key } & \text { Key Integer deriving } & \text { (Show,Eq, Ord) } \\
\text { newtype Name } & \text { Name String deriving } & \text { (Show,Eq) } \\
\text { data Breed } & =\text { Cow } \mid \text { Sheep deriving } & \text { (Show,Eq) } \\
\text { newtype Price } & \text { Price Float deriving (Show,Eq, Ord) } \\
\text { data Disease } & \text { BSE | FM } & \text { deriving (Show,Eq) } \\
\ldots
\end{array}
$$

We derive Show, Eq, and Ord instances to allow for printing of query results, and comparison of cells in WHERE conditions. We redefine Cell such that it is complete for the 'foot-n-mouth' database.

$$
\begin{aligned}
& \text { data Cell }=\text { KeyCell Key } \\
& \mid \begin{array}{lll}
\text { NameCell Name } \\
\text { BreedCell Breed } \\
\ldots & -- \text { and certainly more cases }
\end{array}
\end{aligned}
$$

\section{The universal universe}

Rather than introducing problem-specific universes of cell types, we can employ dynamics [2, 3]. Haskell's library Data.Dynamic provides the type Dynamic and an injection toDyn as well as a projection fromDynamic. Although this approach does not seem more typed, at least it is more extensible: we can make each new userdefined type amenable to injection and projection by providing an instance of Haskell's type class Typeable. There is a fully equivalent alternative: we can use existentially quantified cell types together with a nominal, extensible, type-safe cast [19].

Using dynamic typing, the encoding of column names in cell types allows us to leave them out in the type of query results. A row ends up being a heterogeneous list of Dynamics. That is:

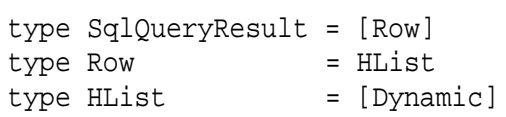

Using injection we construct an HList-typed value for cow Angus:

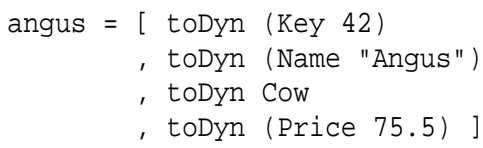

We can process such HLists with ordinary list-processing functions, e.g., head, tail, null, and foldr. We can also provide type-based operations, e.g., an operation hoccursmany to retrieve all elements of a given type: 1

$$
\begin{aligned}
\text { hoccursmany } & : \text { Typeable a }=>\text { HList }->\text { [a] } \\
\text { hoccursmany } & =\text { map fromJust }-- \text { unwrap Just } \\
& \text { - filter isJust }-- \text { remove Nothing } \\
& \text { - map fromDynamic }-- \text { get out of Dynamic }
\end{aligned}
$$

For instance, we can attempt to look up the breed of cow Angus:

$$
\text { ghci-or-hugs> hoccursmany angus : : [Breed] }
$$$$
\text { [Cow] }
$$

Note that printing HLists such as angus requires extra effort. A value of type Dynamic is normally opaque. We can revise toDyn to include a Show constraint in addition to the Typeable constraint. Alternatively, we can provide a Show instance for Dynamic, which attempts frombynamic towards all showable types that we can possibly think of. These two options account for weak extensibility.

\section{Too few or too many types}

Most programmers are likely to loath operating on strings: it is completely untyped. A non-Haskell programmer might regard tagged unions as reasonably typed. The Haskell programmer will ask for much more typing. Most notably, the above type-based look-up gives no static guarantee that an element of the relevant type will be found at run-time.

In database programming, these guarantees correspond to static checks on column access in WHERE phrases and elsewhere. Static checks would require a mapping of the data dictionary to Haskell types. For example, we could define one newtype per database table, with each newtype describing table columns as a product or a record. We can process values of these newtypes with generic functions [19]. However, we are stuck: it is not enough to have precise types for database tables. We also would need precise types for queries and their intermediate expressions. So we face the need for an open-ended set of product or record types. This challenge is addressed below.

${ }^{1}$ To avoid confusion, we prefix all heterogeneously typed functions, types, and classes with an "h" (or, an "H") such as in hoccursmany and HList. 


\section{Typeful heterogeneous lists}

We seek a notion of heterogeneous lists that is more typeful than [Dynamic]. The type of a list should precisely describe the types of its elements, as a type sequence or product. This will allow us to make static promises, e.g., a guarantee that a look-up operation for a type delivers a result. As we will see, precision of typing does not impair our ability to define 'normal' list-processing functionality.

\section{Heterogeneous list constructors}

We start by defining datatypes for lining up type sequences:

$$
\begin{array}{llll}
\text { data HNil } & \text { HNil } & \text { deriving (Eq, Show, Read) } \\
\text { data HCons e } 1 \text { HCons e } 1 & \text { deriving (Eq, Show, Read) }
\end{array}
$$

These datatypes reify normal list structure at the type level, and thereby they allow us to statically distinguish empty and non-empty lists just as in dependently typed programming $[12,21]$. Furthermore, each list element may have a different type.

For less parentheses, we assume right-associative infix operators:

$$
\begin{aligned}
\text { type e }:{ }^{*}: 1 & =\text { HCons e } 1-- \text { type level constructor } \\
e^{*} \cdot 1 & =\text { HCons e } 1-- \text { value level constructor }
\end{aligned}
$$

Here is a type sequence for animals:

type Animal =

Key $:{ }^{\star}$ : Name $:{ }^{\star}$ : Breed $:{ }^{\star}$ : Price $:{ }^{\star}$ : HNil

Here is a heterogeneous list that represents cow Angus:

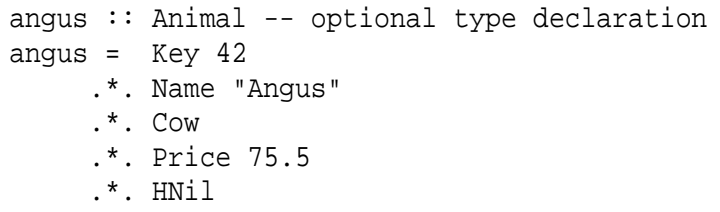

We note that heterogeneous lists are essentially nested tuples. So we could use the normal type constructors () and (,) instead of HNil and HCons as in: (Key, (Name, (Breed, (Price, ())))). We favour fresh datatypes for building heterogeneous list. This helps avoiding confusion and clashes with 'normal' applications of () and $($,$) . We could also consider implicitly terminated type se-$ quences. Again, we require a terminating HNil to avoid a mess.

\section{A class of heterogeneous lists}

When using HCons such as in HCons e 1 , we want the tail 1 to be a heterogeneous list type again. To this end, we will now work out a class HList whose extension is the set of all proper type sequences, i.e., the set of all nested, right-associative, binary products. This class replaces the type [Dynamic] from the previous section.

class HList 1

instance HList HNil

instance HList $1 \Rightarrow$ HList (HCons e l)

What is the purpose of this class? Some readers might wonder whether we want to constrain the type constructor HCons like that:

data HList $1 \Rightarrow$ HCons e 1 = HCons e 1 deriving ...

After due discussion we decided: NO, being in good company [27]. The problem with constraints on datatypes is that they only imply a proof obligation, but type inference does not propagate them nicely. This would lead to a proliferation of HList constraints.

We rather place HList constraints on list-processing functionality whenever we want them. A user of the HLIST library does not employ the unconstrained constructor HCons, but only a constrained version of it. To this end, we retype $\left(.{ }^{\star}.\right)$ :

$$
\begin{aligned}
& \left({ }^{\star} .\right):: \text { HList } 1 \Rightarrow \text { e } \rightarrow 1 \rightarrow \text { HCons e } 1 \\
& \left({ }^{\star} .\right)=\text { HCons }
\end{aligned}
$$

\section{List-processing operations}

Functions on normal lists (e.g., head, tail, and null) can be systematically transposed to the type level. Normally, each type-level operation is subject to a dedicated class; see App. A for some examples, and the HLIST source distribution for additional examples. Let us consider the recursive function for concatenation in some detail. For comparison, we recall normal list concatenation:

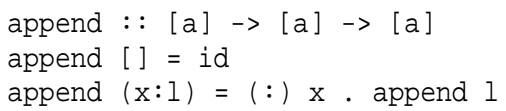

We define a class HAppend for concatenation of heterogeneous lists:

class HAppend $1 l^{\prime} l^{\prime \prime} \mid 1 l^{\prime}->l^{\prime \prime}$

where hAppend : : $1 \rightarrow l^{\prime} \rightarrow 1^{\prime \prime}$

Here we use Haskell's extensions for multi-parameter classes and functional dependencies - which, incidentally, were introduced for the sake of 'normal' collection libraries. So it is not surprising that we end up using these extensions for heterogeneous collections. The functional dependency $l l^{\prime} \rightarrow l^{\prime \prime}$ indicates that the class is a type-level function - rather than a mere relation on types.

The instances follow the definition of append very closely:

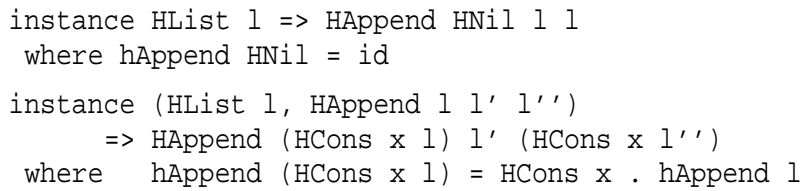

We note that append's equational term patterns show up twice in the class HAppend: once in the instance heads of HAppend and once in its method definitions. Also, the instance constraints for HList are like type checks to be performed at type checking 'run-time'. But otherwise we transcribe list processing to the heterogeneous case in a systematic manner. There is just a constant factor of noise.

Rather than defining all kinds of specific list-processing functions, one might wonder if the general recursion schemes for list processing can also be transcribed to the heterogeneous situation. This is indeed the case; see App. B for a heterogeneous fold operation and the HLIST source distribution for further higher-order operations on HLists.

\section{Aside: stanamic lists}

The class HList, and all the classes with list-processing operations (e.g., the shown HAppend) are in no way restricted to lists built from HNil and HCons. We can easily add instances for HList, HAppend, and others such that we also deal with less typeful heterogeneous lists (e.g., [Dynamic]), or with less generic heterogeneous lists (such as hand-made universes). This allows us to use our collection framework even in cases when the precise type sequence for a collection is not known statically, e.g., when collections are built from user input. One can even mix statically and dynamically typed collections. An advanced example of such a "stanamically" constrained data structure are the balanced trees in [18]. For the rest of the paper we will focus on statically typed heterogeneous lists.

\section{Numeral-based access operations}

We will now define array-like (or numeral-based) access operations for HLists. That is, we will use type-level naturals to address list elements. These access operations provide a basic layer in the HList library because type-based and label-based access operations can actually be implemented in terms of numeral-based operations. 


\begin{tabular}{|c|c|c|c|c|c|c|c|c|}
\hline class & HNat $\mathrm{n}$ & $\Rightarrow$ HLookupByHNat & $\mathrm{n} 1 \mathrm{e}$ & n 1 & 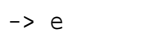 & where & hLookupByHNat & $:: \mathrm{n} \rightarrow 1 \rightarrow \mathrm{e}$ \\
\hline class & HNat $\mathrm{n}$ & $\Rightarrow$ HDeleteAt HNat & $\mathrm{n} 11^{\prime}$ & n 1 & $->1^{\prime}$ & where & hDeleteAtHNat & $:: \mathrm{n} \rightarrow 1 \rightarrow 1^{\prime}$ \\
\hline class & HNat $\mathrm{n}$ & $\Rightarrow$ HUpdateAt HNat & $\mathrm{n}$ e $1 l^{\prime}$ & $\mathrm{n}$ e 1 & $->1^{\prime}$ & where & hUpdateAtHNat & $:: \mathrm{n} \rightarrow \mathrm{e}->1 \rightarrow 1^{\prime}$ \\
\hline class & HNats ns & $\Rightarrow$ HProjectByHNats & ns $11^{\prime}$ & ns 1 & $->1^{\prime}$ & where & hProjectByHNats & $::$ ns $\rightarrow 1 \rightarrow 1^{\prime}$ \\
\hline
\end{tabular}

Figure 1. Numeral-based access operations for heterogeneous collections

\section{Type-level naturals}

Type-level naturals are represented by datatypes for zero and successor function. These datatypes are solely for the type-level: the only value of these types is $\perp .^{2}$

class HNat $n$

data HZero; instance HNat HZero

data HSucc $\mathrm{n}$; instance HNat $\mathrm{n} \Rightarrow \operatorname{HNat}$ (HSucc $\mathrm{n}$ )

hZero : : HZero; hZero $=\perp$

hSucc :: HNat $\mathrm{n} \Rightarrow \mathrm{n} \rightarrow$ HSucc $\mathrm{n}$; hSucc h $_{-} \perp$

hPred : : HNat $\mathrm{n} \Rightarrow$ HSucc $\mathrm{n} \rightarrow \mathrm{n}$; hPred $-=\perp$

Eventually, one needs to perform all kinds of operations on typelevel naturals such as arithmetics or comparison. As an example, we present (type-level) equality, as needed elsewhere in the paper.

First, we need type-level Booleans:

class HBool $x$

data HTrue; instance HBool HTrue

data HFalse; instance HBool HFalse

hTrue :: HTrue; hTrue $=\perp$

hFalse :: HFalse; hFalse $=\perp$

-- classes for HAnd and HOr omitted

Here are the classes for general type-level equality and comparison including the straightforward instances for the equality of naturals:

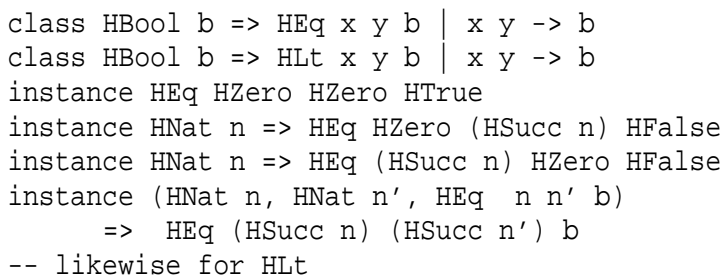

\section{Induction on type-level naturals}

One can define various access operations using naturals as indices; see Fig. 1 for an overview. For instance, the delete operation boils down to two instances: one for HZero; another for HSucc:

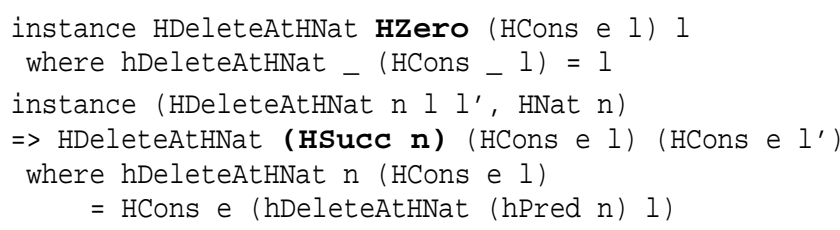

\section{Extra constraints}

Functionality on collections carries implied constraints due to all the involved access operations. In addition, one might want to add extra constraints. For instance, we can use the following class to restrict the maximum length of a list (or an array):

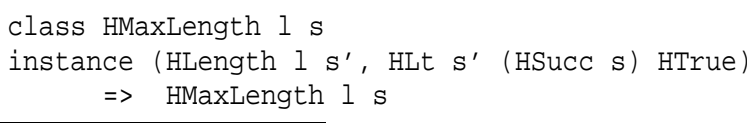

${ }^{2}$ We also prefix all faked dependently typed functions and types with an "h" (or, an "H") such as in hTrue and HBool. These types correspond to subsets of values of normal types such as Int, and so let us discriminate the subsets of values at compile time.

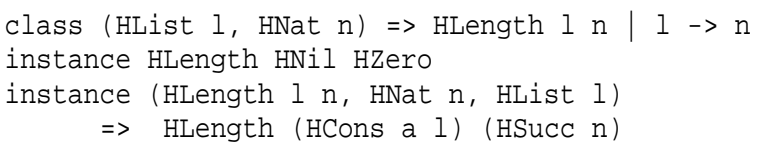

By adding HMaxLength constraints to signatures or instances, one instructs Haskell to enforce size boundaries at compile time.

\section{Extensible records}

We will now define labelled collections, i.e., maps from labels to values. In essence, we will employ type-level naturals for labels, but we will enrich the structure of labels for convenience of programming with labelled collections. We end up defining extensible records this way, without requiring the language extensions of earlier proposals. From the point of view of database access, records provide the ultimate expressiveness for mapping column names to values in a typeful manner. Extensibility (and shrinkability) of records is key to dealing with types of joins and projections.

\section{Haskell's nonextensible records recalled}

In Haskell 98, we can define record types like this:

$$
\begin{aligned}
\text { data Unpriced }=\text { Unpriced } & \{\text { key }: \text { Integer } \\
& , \text { name : : String } \\
& , \text { breed }: \text { : Breed }\}
\end{aligned}
$$

Here is a unpriced cow Angus:

$$
\begin{aligned}
& \text { unpricedAngus = Unpriced }\{\text { key }=42 \\
& \text {, name = "Angus" } \\
& \text {, breed = Cow }
\end{aligned}
$$

What are access operations that are available for Haskell 98 records? We can retrieve components, and we can update records in a point-wise fashion:

$$
\begin{aligned}
& \text { ghci-or-hugs }>\text { breed unpricedAngus } \\
& \text { Cow } \\
& \text { ghci-or-hugs }>\text { unpricedAngus }\{\text { breed = Sheep }\} \\
& \text { Unpriced }\{\text { key }=42, \text { name="Angus", breed=Sheep }
\end{aligned}
$$

We can not extend such records (unless we were thinking of nesting records and using polymorphic dummy fields for extension [6]). Also, we can not reuse labels among different record types, neither can we treat labels as data; so labels are not first-class citizens.

\section{An extensible record demo}

We place related labels in a namespace modelled by a silly datatype: data FootNMouth = FootNMouth -- a namespace

Labels in a namespace are constructed in a sequence starting with firstLabel, with next Label generating the next distinguished label. Each label is also annotated with a string for the label name. These are the labels for animals:

$$
\begin{array}{llll}
\text { key } & =\text { firstLabel } & \text { FootNMouth "key" } \\
\text { name } & =\text { nextLabel } & \text { key } & \text { "name" } \\
\text { breed } & =\text { nextLabel } & \text { name } & \text { "breed" } \\
\text { price } & =\text { nextLabel breed } & \text { "price" }
\end{array}
$$


We build the record for the unpriced cow Angus as follows:

$$
\begin{aligned}
& \text { unpricedAngus }=\text { key } \quad .=.(42:: \text { Integer }) \\
& .{ }^{*} \text {. name } .=\text {. "Angus" } \\
& { }^{*} \text {. breed } .=\text {. Cow } \\
& \text {.* . emptyRecord }
\end{aligned}
$$

That is, record construction starts from emptyRecord; the labelvalue pairs are connected by ".=."; and each label-value pair is added by using an overloaded operation ". *.”.

Extensible records are printed more or less like Haskell 98 records:

ghci-or-hugs $>$ unpricedAngus

Record $\{$ key $=42$, name="Angus" , breed=Cow $\}$

We retrieve a component from a record as follows:

ghci-or-hugs> unpricedAngus .!. breed

Cow

We can update components as follows:

ghci-or-hugs $>$ unpricedAngus .@. breed .=. Sheep

Record $\{$ key=42, name="Angus", breed=Sheep $\}$

We can really extend such records:

ghci-or-hugs $>$ price.$=.8 .8 .{ }^{\star}$. unpricedAngus

Record $\{$ price $=8.8$, key $=42$, name $="$ Angus" , breed $=$ Cow $\}$

\section{One possible model of extensible records}

Labels can be implemented by type-level naturals, qualified by a namespace, and annotated by a string for the label name:

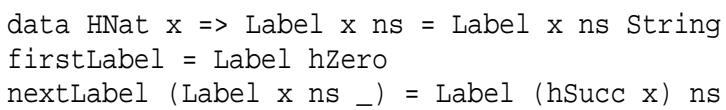

Records are maps from labels to values. We could go for heterogeneous lists of pairs; we could also go for pairs of heterogeneous lists of equal length. We abstract from this choice as follows:

$$
\begin{aligned}
& \text { Class HZip x y } 1 \mid x y \rightarrow 1,1 \rightarrow x y \\
& \text { where hZip : : x } \rightarrow \text { y } \rightarrow 1 \\
& \text { hUnzip : : } 1 \rightarrow(x, y)
\end{aligned}
$$

A record is a zipped list wrapped within Record:

newtype Record $r=$ Record $r--$ to be constrained

Record construction is constrained as follows:

$$
\begin{aligned}
\text { mkRecord } & :: \text { (HZip ls vs } r \text {, HLabelset ls) } \\
& \Rightarrow r \rightarrow \text { Record } r
\end{aligned}
$$

mkRecord $=$ Record

For instance, the empty record is denoted as follows:

emptyRecord $=$ mkRecord $\$$ hZip HNil HNil

Labels in a record must be distinct:

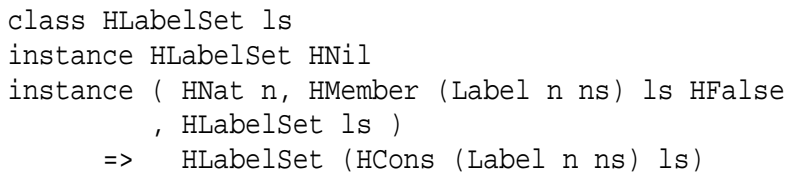

To this end, we define HEq-based membership test as follows:

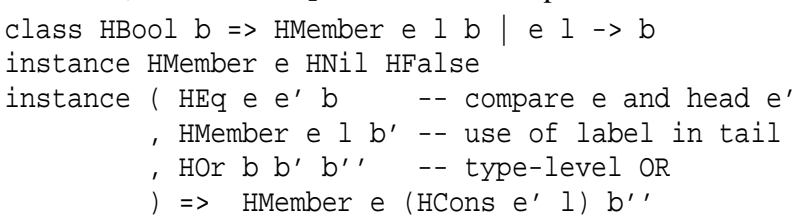

We also extend equality, which was already defined for type-level naturals, such that we can compute equality of labels. Here we assume that the labels in a record are in the same namespace:

instance HEq $x x^{\prime}$ b-- compare naturals in labels

$\Rightarrow$ HEq (Label $x$ ns) (Label $x^{\prime} n s$ ) b

\section{Access operations}

In the demo, we encountered access operations for look-up, update, and extension. There are also operations for appending records, for deletion of a label and its value in a record, for renaming of a label in a record, for projection and splitting of a record according to a label set. We can implement these operations directly on the representation of records (cf. "pair of lists" vs. "list of pairs"). Alternatively, we can use numeral-based access complemented by zipping and unzipping.

For instance, deletion (“.-.") can be defined as follows:

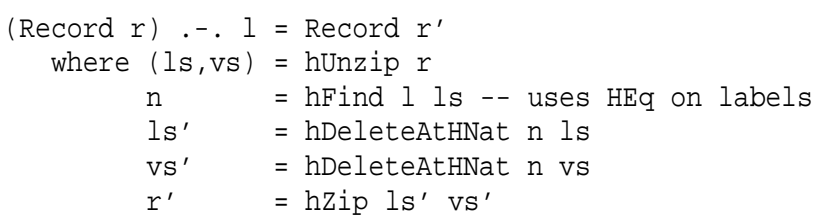

That is, we unzip the record; we find the index $n$ of the given label $I$ in the list Is of labels; we delete the subscripted elements in the lists is and vs of labels and values; we finally re-zip the record.

\section{Type-based access operations}

Numeral-based and label-based access is in some sense still valuebased - even though we had to reify naturals at the type level. We will now work out truly type-based access operations. From a database perspective, type-based operations are useful when types are descriptive of columns. In that case, there is no need to employ label-to-value mappings.

As for the coding style, we will make transient use of overlapping instances, as supported by the GHC and Hugs implementations of Haskell. We later circumvent overlapping instances.

\section{Filter an HList for elements of a given type}

The operation hoccursmany from Sec. 2 is an example of a typebased operation. The type of elements to be extracted from a list of dynamics is specified by fixing the result type of hoccursmany. We will now define such type-based operations on HList including more strongly typed ones; see Fig. 2 for an overview.

We dedicate a class to hoccursmany:

class Hoccursmany e 1 where hoccursmany :: 1 -> [e]

The instance for HNil returns []:

instance HOccursMany e HNil where hOccursMany _ = [] Another instance deals with a non-empty HList whose head is of the type of interest; notice that $e$ is used twice in the instance head:

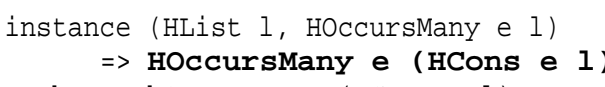

where hoccursmany (HCons e l) = e : hoccursmany 1

There is yet another instance for a non-empty HList whose head is not of the same type as the element type in hoccurs's result type:

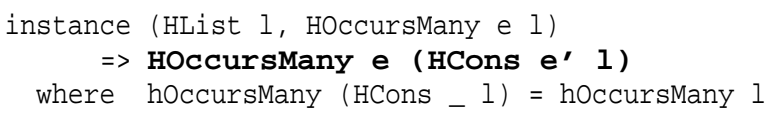

The two HCons instances are overlapping, while the former is more specific than the latter, which is thereby only applied when the former is not applicable, i.e., whenever the types e and $e^{\prime}$ are different. hoccursmany is the regular "*" operation for type-based lookup. Then there are similar operations hoccursmany1 (i.e., “+”), hOccursOpt (i.e., "?”), and hOccursFst (for the first occurrence). The class HOccurs and its complement HOccursNot require more thought. Most notably, a type-checked application of hoccurs is supposed to assure that there is exactly one element of the type in 


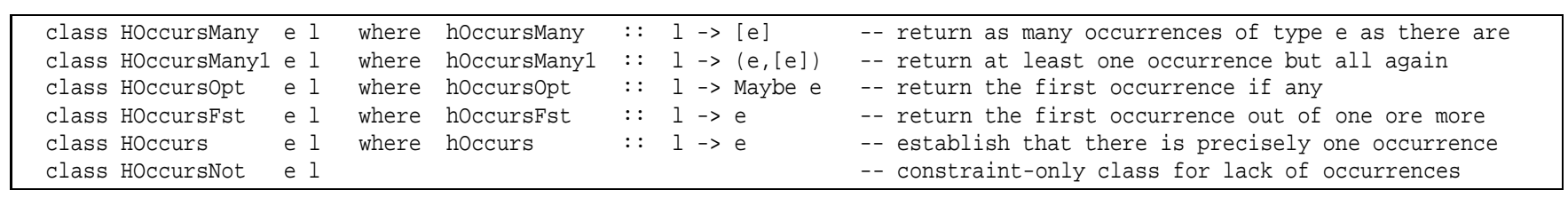

Figure 2. Type-based look-up operations for heterogeneous collections

question. Successful type checking of hoccurs angus :: Breed implies that angus's breed is defined unambiguously. We will develop the definitions of HOccurs and HOccursNot in detail.

\section{Documenting potential type errors}

At first sight, there is no HOccurs instance for HNil, but we can provide one - be it for the sake of instructive error messages. Instances like the following make class-based dependently typed programming more manageable:

$$
\begin{aligned}
& \text { instance Fail (TypeNotFound e) } \Rightarrow \text { HOccurs e HNil } \\
& \text { where hoccurs }=\perp
\end{aligned}
$$

Here we use a vacuous class Fail without instances, which just implements what its name promises, and we also assume a datatype TypeNotFound that serves for nothing but an error message:

$$
\text { class Fail x -- no methods, no instances! }
$$$$
\text { data TypeNotFound e -- no values, no operations! }
$$

Hence we obtain somewhat suggestive error messages:

$$
\text { ghci-or-hugs > hoccurs (HCons True HNil) : : Int }
$$$$
\text { No instance for (Fail (TypeNotFound Int)) }
$$

So we try to look up a value of a type that's not in the list. Hence, iteration ends up at HNil, and TypeNotFound is reported. Such documentary failure instances are used throughout the HList library.

\section{Static look-up}

We will now provide the actual definition of hoccurs. There are again two overlapping instances for non-empty lists; one for the case that the head fits with the type of interest, and another for recursion in case we haven't found an occurrence yet:

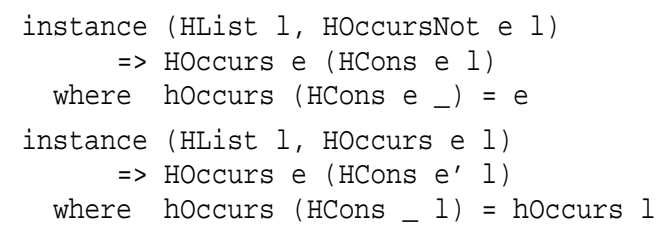

The constraint HOccursNot e 1 in the first instance assures that no elements of type e occur in the tail 1 . The class HOccursNot is for constraining only rather than actual look-up. Consequently, its definition does not comprise any method:

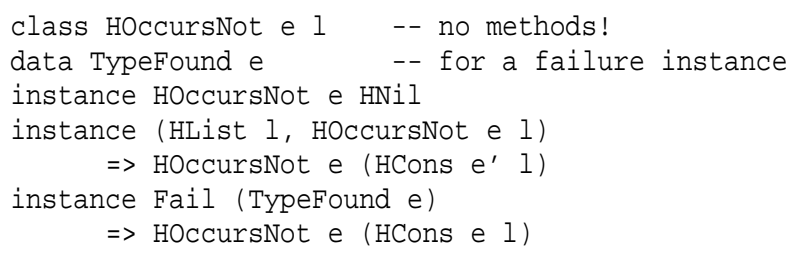

The instances fold over $l$ to test that each type is different from e. The last instance leads to failure for an offending head. This failure instance is obligatory because the more general instance for HCons would otherwise silently skip over the offending occurrence. Notice that Haskell's instance selection is solely based on syntactical matching. Hence, the failure of the more specific instance (via Fail) will not lead to reconsideration of the more general instance.

\section{From look-up to projection}

We can now readily define projection by mapping over a list of requested element types using simple look-up for each element type; see the HLIST source distribution for the actual code. For instance, the following query retrieves the key and the name of cow Angus:

$$
\begin{aligned}
& \text { ghci-or-hugs }>\text { hProject angus } \\
&: \text { : (HCons Key (HCons Name HNil)) } \\
& \text { HCons (Key 42) (HCons (Name "Angus") HNil) }
\end{aligned}
$$

This operation resembles projection in the sense of relational algebra, or in the sense of SQL'S SELECT statements. (Think of the column names following the keyword SELECT.)

\section{Type-based mutation operations}

We also need mutation operations such as the following:

- Delete list elements identified by their type.

- Update list elements by values of the same type.

- Split a list into a projected list and its complement.

The update operation(s) mutate at the value level only, e.g.:

$$
\begin{aligned}
& \text {-- Replace the occurrences of type e } \\
& \text { class HUpdateMany e } 1 \\
& \text { where hUpdateMany : : e } \rightarrow 1->1
\end{aligned}
$$

So the type-level programming bits of look-up can be adopted for type-preserving update. Deletion requires functional dependencies: -- Delete the occurrences of type e in 1 , return $1^{\prime}$ class HDeletemany e $l^{\prime} \mid$ e $l \rightarrow l^{\prime}$

where hDeleteMany :: ... -- to be completed

Such mutation operations also mutate types. Without functional dependencies, users had to specify the result type explicitly, which is impractical. The trouble is that the combination of overlapping instances and functional dependencies leads us into murky water. We take this as an incentive to identify an overlapping-free idiom.

\section{Passing on types as proxies}

Let us first get the type of hDeleteMany right. It could be this one: class HDeleteMany e $1 l^{\prime} \mid$ e $1 \rightarrow l^{\prime}$

The argument of type e would merely describe the type of the elements that should be deleted. We might not have any suitable value around (except $\perp$ ). Also, the above type obscures the role of the first argument. So we go for this type instead:

hDeleteMany :: Proxy e $\rightarrow 1->1^{\prime}$

Proxies are defined as follow:

data Proxy e; proxy :: Proxy e; proxy $=\perp$

Hence, the only value of a proxy type is the specific value $\perp$ of the constructed proxy type - not to be confused with the value $\perp$ of the type being proxied. We can reduce values to proxies if needed:

toProxy : : e $\rightarrow$ Proxy e; toProxy $\_=\perp$

For example, we delete the name of cow Angus as follows:

ghci-or-hugs> hDeleteMany (proxy: :Proxy Name) angus HCons (Key 42) (HCons Cow (HCons (Price 75.5) HNil)) 


\section{A non-solution}

Adopting the style that we offered for look-up operations, we would want to implement hDeleteMany with one instance for HNil; one instance for 'delete head'; one instance for 'keep head':

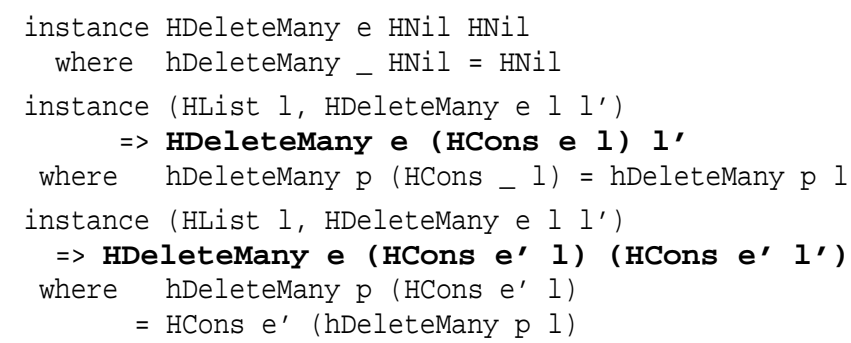

Alas, the two overlapping instance heads for HCons are in no substitution ordering. (Neither GHC nor Hugs can be persuaded to accept this code.)

\section{Move patterns from the head to constraints}

There is a rescue. We simply need to generalise one instance head so that it becomes more general than the other. Then, instance selection will be re-enabled. We generalise the head of the last instance:

- before: HDeleteMany e (HCons $e^{\prime}$ l) (HCons $e^{\prime} 1^{\prime}$ )

- after: HDeleteMany e (HCons $e^{\prime} 1$ ) $1^{\prime \prime}$

But we must maintain the type equation $l^{\prime \prime}$ equals HCons $e^{\prime} l^{\prime}$ ! To this end, we employ type cast. We add an instance constraint TypeCast (HCons $\mathrm{e}^{\prime} \mathrm{l}^{\prime}$ ) $\mathrm{l}^{\prime \prime}$, and we also cast in the method:

instance ( HList l, HDeleteMany e 1 l'

$$
\begin{aligned}
& \text { ' TypeCast (HCons } \left.e^{\prime} l^{\prime}\right) l^{\prime \prime} \text { ) } \\
& \Rightarrow \text { HDeleteMany e (HCons } e^{\prime} 1 \text { ) } 1^{\prime \prime} \\
& \text { where hDeleteMany } \mathrm{p} \text { (HCons } \mathrm{e}^{\prime} \mathrm{l} \text { ) } \\
& \left.=\text { typeCast (HCons } \mathrm{e}^{\prime} \text { (hDeleteMany } \mathrm{p} 1\right) \text { ) }
\end{aligned}
$$

There is no shortage of type-safe casts for Haskell [34, 8, 4, 19]. The one we need here is really resolved at the type-level. So there is no Maybe involved, since typeCast cannot fail at run-time:

$$
\begin{aligned}
& \text { class TypeCast } \mathrm{x} y \mid \mathrm{x} \rightarrow \mathrm{y}, \mathrm{y} \rightarrow \mathrm{x} \\
& \text { where typeCast }: \mathrm{x} \rightarrow \mathrm{y}
\end{aligned}
$$

The functional dependencies capture our expectation of type cast to be an isomorphism on types (in fact, the identity function). We will discuss the implementation of TypeCast in Sec. 9.

\section{Ended up in murky water}

There is no real consensus on the overlapping instance mechanism as soon as functional dependencies are involved. Our result from above fits with GHC's model, but Hugs reports that the instances are inconsistent with the functional dependency for HDeleteMany. Here is a simple example that exercises this disagreement:

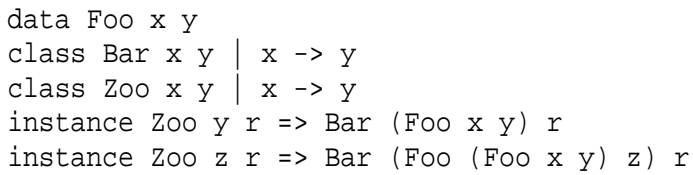

Hugs' type system misses the point that Bar's second parameter is still functionally dependent on part of Bar's first parameter.

\section{Overlapping banned}

We give up on persuading Hugs. Also, we do not want to depend on the doubtful future of overlapping instances in general. Furthermore, regimes for instance selection differ in ways other than consistency criteria for functional dependencies. For instance, GHC's instance selection is lazy, whereas Hugs' is eager.
We avoid overlapping instances by reformulating our problem into a case selection driven by a type-level Boolean denoting a computed type equality. The predicate for type equality is provided as follows:

class HBool $\mathrm{b} \Rightarrow$ TypeEq $\mathrm{x}$ y $\mathrm{b} \mid \mathrm{x}$ y $\rightarrow \mathrm{b}$

proxyEq :: TypeEq $t t^{\prime} b \Rightarrow$ Proxy $t \rightarrow$ Proxy $t^{\prime} \rightarrow b$ proxyEq $-{ }_{-}=\perp$

We take for granted that we can define type equality; see Sec. 9. Using type equality, we replace the overlapping instances for HDeleteMany by the following case-preparing instance:

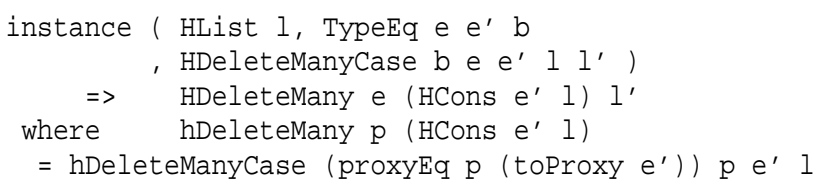

That is, we compute type equality so that we are able to decide whether the head needs to be deleted. This decision is then implemented by the helper class HDeleteManyCase with instances (i.e., branches) for the two Booleans:

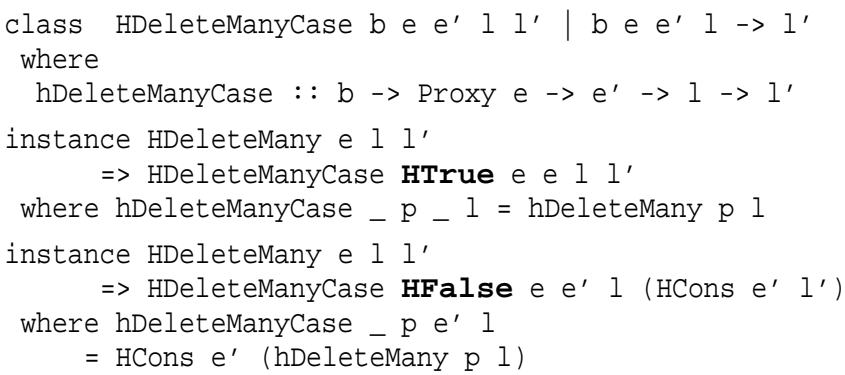

This idiom works equally well for other type-based operations.

\section{Type-to-natural mapping}

We can even factor out case discriminations for type equality to be used in just a single location, namely in a type-to-natural mapping. The remaining type-based access operations can then employ this mapping completed by numeral-based access.

The type-to-natural mapping is hosted by the following class:

class HNat $\mathrm{n} \Rightarrow$ HType2HNat e $1 \mathrm{n} \mid$ e $1 \rightarrow \mathrm{n}$

The implementation adopts the overlapping-free idiom:

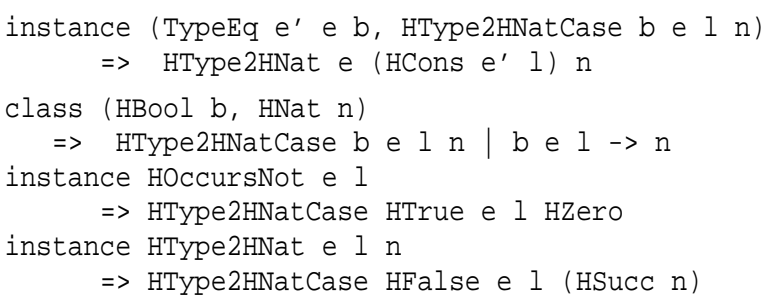

We note that the first instance carries a constraint HOccursNot e 1 . This makes sure that the type e in question is associated with a single natural as index. Alternatively, we could return a list of a indexes for elements of type e. This would be necessary for the reconstruction of operations like hoccursmany.

For instance, type-based delete can now be expressed concisely in terms of numeral-based delete - without the hassle of a helper class for case discrimination on Booleans:

hDelete p 1 = hDeleteAtHNat (hType2HNat p l) 1

Here we invoke the type-to-natural mapping using this function:

hType2HNat : : HType2HNat e $1 \mathrm{n} \Rightarrow$ Proxy e $\rightarrow 1 \rightarrow \mathrm{n}$ hType2HNat $-{ }_{-}=\perp$ 


\section{Aside: type schemas and class-based programming}

The fine details of our heterogeneous collections reflect the employment of Haskell's class concept. Most notably, all involved type schemas must be sufficiently instantiated to allow for instance selection without causing ambiguities. This is just the same as in the case of show . read whose application to a string cannot be evaluated because the type of the intermediate result is not fixed.

We can store and look up polymorphic values as long as their type schemas are not needed for instance selection. So numeral-based access works fine even for arbitrary polymorphic elements, because the element types do not drive instance selection:

ghci-or-hugs $>$ hLookupByHNat hzero (id .*. HNil) \$ 42 42

The following type-based access still works:

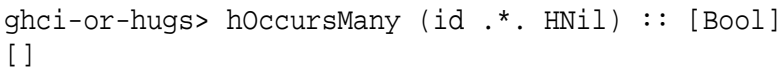

We note that hoccursMany compares its result type with all element types. The type schema forall a. a $\rightarrow$ a of id is sufficiently instantiated for that, i.e., forall a. a $\rightarrow$ a is different from Bool for all possible a. Here is an example of an ambiguous situation:

ghci-or-hugs $>$ hoccursmany $\left(\perp .{ }^{*}\right.$. HNil) : : [Bool]

No instance for ... <snipped>

The interaction of polymorphic elements in collections and classbased programming will continue to be a topic in the next section.

\section{Type-indexed products}

As a refinement of type-based access to heterogeneous collections, one can even require that a given collection is entirely type-indexed, i.e., that no type occurs more than once. Imposing this requirement on lists, we obtain so-called type-indexed products (TIPs; [31]). We will now briefly describe an implementation of TIPs. The dual of TIPs, TICs, are defined in App. C.

We wrap TIPs in a newtype so that we make the status of being typeindexed explicit in type signatures. Also, we can provide special instances for TIPs once we made this type distinction:

newtype $\operatorname{TIP} 1=\operatorname{TIP} l_{--}$to be constrained

unTIP $($ TIP 1$)=1$

The public constructor for TIPs supplies the key constraint for TIPs:

mkTIP : : HTypeIndexed $1 \Rightarrow 1->\operatorname{TIP} 1$

$\operatorname{mkTIP}=\operatorname{TIP}$

The class HTypeIndexed is defined as follows:

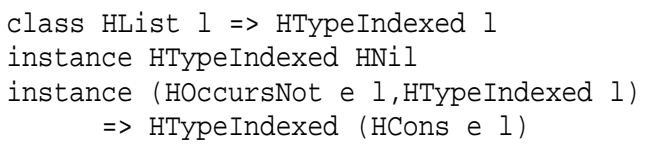

The instances traverse over the type sequence, and the class HOccursNot is employed to assure that the type of the head does not occur (again) in the tail.

Let us upgrade angus to a TIP:

ghci-or-hugs $>$ let myTipyCow = TIP angus

\section{Lifting operations}

Most trivially, there is a replacement for HNil:

emptyTIP = mkTIP HNil

Operations on TIPs are lifted as follows. "TIP" is unwrapped in arguments, and it is wrapped in the result (if this is a TIP), while constraints are added so that the HTypeIndexed property is enforced. For instance: instance (HAppend $l l^{\prime} l^{\prime \prime}$, HTypeIndexed $l^{\prime \prime}$ )

$\Rightarrow$ HAppend (TIP l) (TIP l') (TIP $\left.l^{\prime \prime}\right)$

where hAppend (TIP 1$)\left(\operatorname{TIP} l^{\prime}\right)=\operatorname{mkTIP}$ (hAppend $l^{\prime} l^{\prime}$ )

Likewise we overload $\left(.{ }^{*}\right.$. ) to work for TIPs, i.e., extensions are assured to preserve the TIP property. To illustrate extension, we label myTipyCow with BSE:

ghci-or-hugs > BSE .*. myTipyCow

TIP (HCOns BSE ...)

The animal myTipyCow is a cow; so it can't be a sheep then:

ghci-or-hugs> sheep .*. myTipyCow

No instance for (Fail (TypeFound Breed))

\section{Subtype constraints}

TIPs naturally give rise to a subtype property. One TIP type $l$ is a subtype of another TIP type $l^{\prime}$ if $l$ contains all types from $l^{\prime}$. This is expressed as follows:

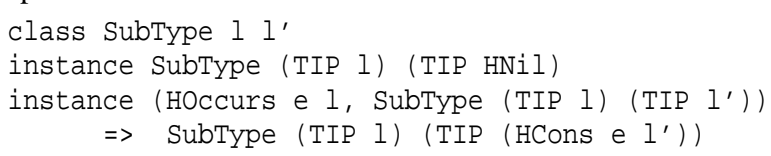

From this it is clear that we do not care about the order of elements in the type-indexed products. We also note that the intersection of HSubType $x y$ and HSubType $y x$ immediately provides a faithful form of type equivalence for TIPs (while mere equality of the underlying type sequences would not be faithful).

As an aside, we can also instantiate subtyping for records. (This can be used in deriving an effective object system in Haskell.) A record type $r$ is a subtype of some record type $r^{\prime}$ if $r$ contains at least the labels of $r^{\prime}$, and the component types for the shared labels are the same. Projection according to label sets is of use here:

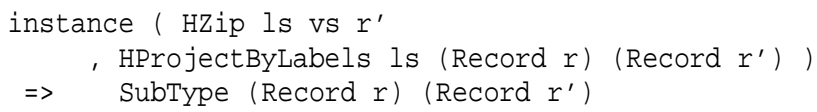

\section{An idiom for constraint annotation}

Let us review idiomatic support for adding extra constraints. For instance, let us deploy a constrained hoccurs that is meant to return the Key of an animalish TIP. TIPs that are not of a subtype of TIP Animal are to be rejected - even if they carry a Key. This can be encoded as follows:

$$
\begin{aligned}
& \text { animalKey :: ( SubType } 1 \text { (TIP Animal) -- extra } \\
& \text {, Hoccurs Key } 1 \text {-- implied } \\
& \text { ) } \Rightarrow 1 \rightarrow \text { Key }
\end{aligned}
$$

The trouble is that this conservative approach forces one to gather all the implied constraints and to make them explicit just as the extra constraints. There is an idiom that allows one to solely enumerate extra constraints. Essentially, one defines a constrained identity function that imposes the constraints of interest on its argument.

The following identity function insists on animals:

$$
\text { animalish :: SubType } 1 \text { (TIP Animal) } \Rightarrow 1 \text {-> } 1
$$

animalish $=$ id

We can now discipline the Key getter as follows: animalkey 1 = hoccurs (animalish 1) : Key The subtype constraint takes action as one can see here:

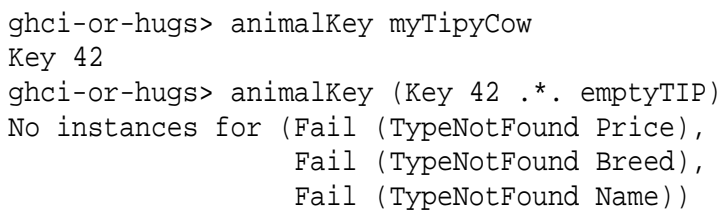


The error message lists the types that are missing from Animal.

\section{A polymorphism benchmark}

As proposed by a reviewer of this paper, we will now consider an example from [31], which is, in a way, about type-based matching.

The following function selects two elements from a collection:

$$
\begin{aligned}
\text { tuple } 1=\text { let } x & =\text { hoccurs } 1 \\
l^{\prime} & =\text { hDeleteAtProxy (toProxy } x) ~ \\
y & =\text { hOccurs } 1^{\prime} \\
\text { in }(x, y) &
\end{aligned}
$$

The following session shows that we can match the elements of a collection in whatever order, while the overloaded operations in tuple are resolved by the consumers of the matched values:

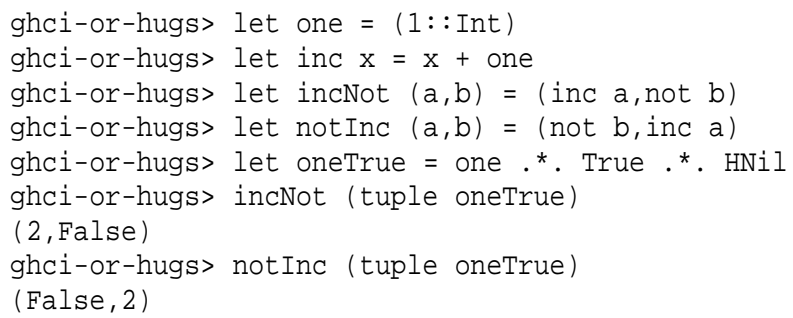

The following example should arguably work, but it doesn't:

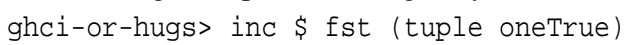

We are going to make this work as well! We note that oneTrue stores two components; so by fixing the type of one component to Int, it should not matter that the type of the other component is left unspecified. The problem boils down to the following issue:

ghci-or-hugs $>$ hoccurs (HCons True HNil)

No instance for (HOccurs e (HCons Bool HNil))

We would like to default e to Bool here. Rather than comparing the type of the head with a not yet instantiated result type, the two types should be unified. The hoccurs operation for TIPs does this:

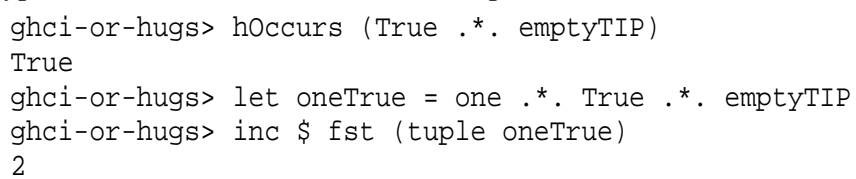

Even the following added polymorphism is handled:

ghci-or-hugs $>$ let oneNull $=$ one $.{ }^{*} .[] . *$. emptytip ghci-or-hugs> inc \$ fst (tuple oneNull) 2

The key idea is to provide a special instance for singleton lists, and to replace the test for type equality by unification via type cast:

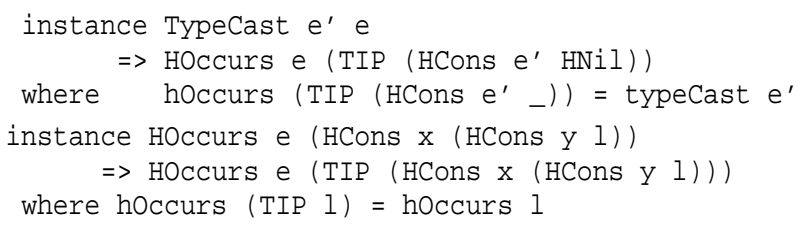

This example reveals that type cast provides a powerful idiom for type improvement - a more fine-grained one than functional dependencies. That is, type cast operates at the instance level as opposed to the class level!

\section{Database programming}

We will now demonstrate heterogeneous collections for database programming in Haskell. To this end, we adopt concepts from Leijen and Meijer's embedding approach for SQL [20]. We employ extensible records for two purposes:
- to represent the results of queries, and

- to represent schemas for relational algebra operations. A detailed discussion of the approach is beyond the scope of this paper. We note however that the approach scales to the full relational algebra, and to a rich set of SQL idioms including all kinds of joins, existential quantification, nested queries, and table statements.

We recall the simple query from the beginning of the paper: SELECT key, name FROM Animal WHERE breed = 'sheep'; In Haskell, we can now write this query in a type-safe manner.

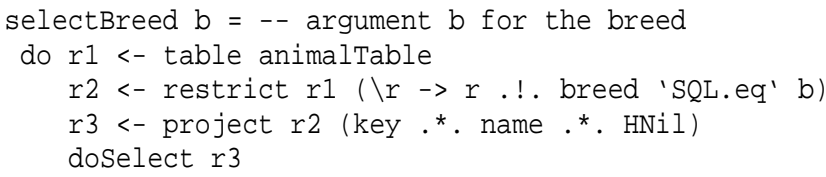

Type inference works fine, but here is the type of the query anyway:

$$
\begin{gathered}
\text { selectBreed : : Breed } \rightarrow \text { Query [ } \\
\text { Tkey :=: Animalld }:{ }^{\star} \text { : } \\
\text { Tname }:=\text { : String }:{ }^{*} \text { : HNil] }
\end{gathered}
$$

That is, the result is a query for records with two components. (The types for the labels key and name are denoted by Tkey and Tname.) The above do sequence encodes the SQL query in four steps:

- r1: We identify the table as in "FROM Animal".

- r2: We restrict the table according to the WHERE condition.

- r3: We perform projection as in "SELECT key, name".

- doselect r3: The actual query is issued.

Steps 1-3 do not involve any database access. (Monadic style is used for hygienic name supply.) The operations table, restrict, project create or modify type-annotated, syntactical expressions for relations. The underlying key data structure looks as follows:

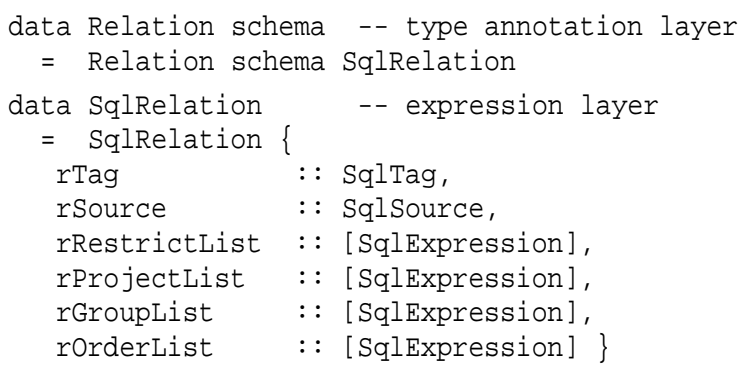

That is, relations carry a schema, and their structural ingredients comprise a unique tag, a source (i.e., a database table), as well as lists of expressions describing restrictions (cf. WHERE), projections (including computed columns), grouping and ordering.

The type of the relational schema for animals is the following:

$$
\begin{aligned}
& \text { type AnimalSchema = } \\
& \text { Tkey :=: Attribute Animalld Sqlinteger : * } \\
& \text { Tname :=: Attribute String SqlVarchar : * } \\
& \text { Tbreed : :=: Attribute Breed SqlVarchar :*: } \\
& \text { Tprice :=: Attribute Float SqlNumeric : * } \\
& \text { Tfarm :=: Attribute FarmId Sqlinteger : }{ }^{\star} \text { : HNil }
\end{aligned}
$$

The schema type lists both the domain of a column and the corresponding SQL type. For instance, the Haskell type for the key component is the newtype Animalid rather than the SQL type Sqlinteger. This 'domain as newtypes' technique increases type safety: one cannot possibly confuse an AnimalId and a FarmId. We note that some of the column types could be wrapped in Maybe, but this is not the case for AnimalSchema.

The datatype Attribute is a phantom type in its two type parameters. These phantoms drive coercions and make attribute access type-safe. For instance, consider the subexpression 
$r$. !. breed 'SQL.eq' $b$ for restriction in the above query. The look-up r. ! . breed does not just establish that there is a breed component, but it also delivers a phantom-typed attribute, so that its use in the compound expression is type-constrained.

Structurally, attributes keep track of some details such as precision, and NULL constraints. All such information is extracted from the data dictionary of a database.

Here is a snippet of the extracted table description for animals:

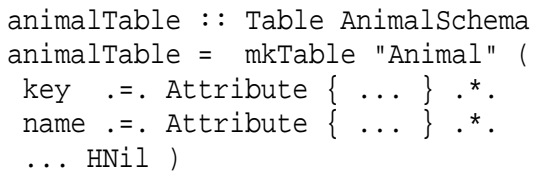

This is all what's needed to make attribute access type-safe. Returning typed query results relies on further provisions. That is, the action doSelect for executing a query has to recast query results such that they are phrased in the Haskell types for column domains.

The code for the execution of SELECTs makes it all clear:

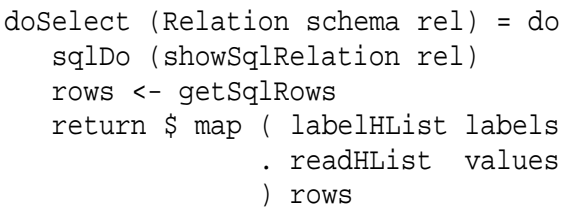

The subexpression showSqlRelation rel computes the SELECT statement as a string, which is then given to sqlDo — the low-level, ODBC-based SQL handler. In the next step, we get all the queried rows as a lazy list of lists using this SQL service:

getSqlRows :: SqlHandle [[Maybe String]]

The subsequent map transforms the string-based rows into typeful ones in two steps. Firstly, we build an HList from the strings with readHList, while we use the attributes from the schema to drive this heterogeneous list construction. Secondly, we turn the HList into a record, while we reuse the labels of the schema.

\section{By chance or by design?}

We will now discuss the issues surrounding the definition of type equality, inequality, and unification - and give implementations differing in simplicity, genericity, and portability.

We define the class TypeEq $x$ y b for type equality. The class relates two types $\mathrm{x}$ and $\mathrm{y}$ to the type HTrue in case the two types are equal; otherwise, the types are related to HFalse. We should point out however groundness issues. If TypeEq is to return HTrue, the types must be ground; TypeEq can return HFalse even for unground types, provided they are instantiated enough to determine that they are not equal. So, TypeEq is total for ground types, and partial for unground types. We also define the class TypeCast $\mathrm{x}$ $y$ : a constraint that holds only if the two types $x$ and $y$ are unifiable. Regarding groundness of $\mathrm{x}$ and $\mathrm{y}$, the class TypeCast is less restricted than TypeEq. That is, TypeCast $x$ y succeeds even for unground types $\mathrm{x}$ and $\mathrm{y}$ in case they can be made equal through unification. TypeEq and TypeCast are related to each other as follows. Whenever TypeEq succeeds with HTrue, TypeCast succeeds as well. Whenever TypeEq succeeds with HFalse, TypeCast fails. But for unground types, when TypeCast succeeds, TypeEq might fail. So the two complement each other for unground types. Also, TypeEq is a partial predicate, while TypeCast is a relation. That's why both are useful.

\section{A representation-based equality predicate}

The predicate TypeEq $x$ y b was introduced in Sec. 6 as follows: class HBool b $\Rightarrow$ TypeEq $x$ y b $\mid x y \rightarrow b$

We now need to provide instances of the class. A very naive implementation would be to explore all combinations of all possible types; see the HLIST source distribution for an illustration. Albeit being portable (Haskell $98+$ multi-parameter classes), this leads to an impractical, exponential explosion in the number of instances. A more scalable approach is to introduce a family of infinite types for type-level type representations. That is, we associate types with type representations via a bijection, and we make sure that type representations are more easily compared than the types themselves. We already have all tools for constructing the family of type representations: we can associate with each type constructor an HNat, and associate with each type term an HList of the representations for the type constructor and its arguments. For instance, using ' 0 ' for Bool, '1' for Int, '2' for $->$, we obtain:

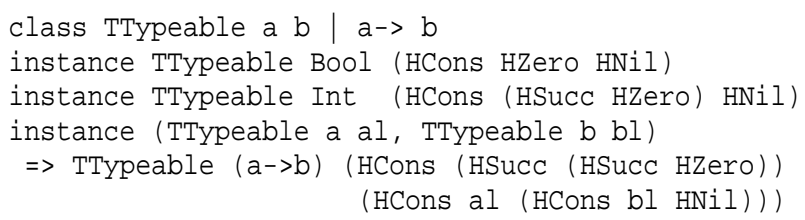

Because these type representations are constructed in a regular way with ever-increasing naturals, it is sufficient to accommodate typelevel equality such that it can compare heterogeneous lists of typelevel naturals. Type-level equality for naturals was given in Sec. 4. Here are the remaining instances for HNil and HCons:

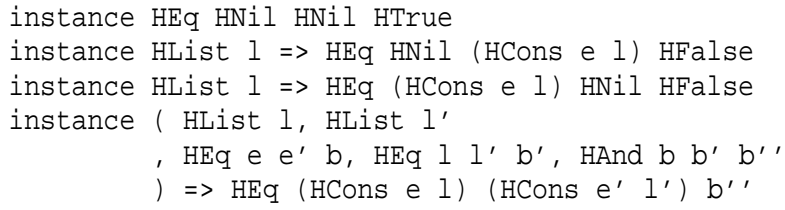

All the involved functionality does not go beyond Haskell 98 and multi-parameter classes with uni-directional functional dependencies. GHC and Hugs readily support this combination.

We can now define the class TypeEq, using the following instance:

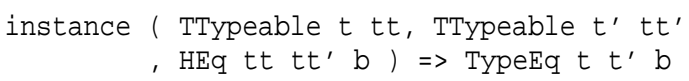

We make use of a generic instance, which is a common Haskell 98 extension. In turns out that we have essentially transposed what's known as the Data. Typeable approach [19] to the type level. We share the drawback of this approach: we need to define an instance of TTypeable for each new type constructor. When adding new instances, we have to maintain the bijection between types and type representations. On the other hand, the remaining code is fully generic and does not need to be amended at all.

\section{A generic type equality predicate}

We have seen that we can implement TypeEq in a portable and even practically usable way, using only commonly supported Haskell extensions. We would like to introduce a fully generic approach, which does not need to be amended when a new type constructor is introduced. Alas, this elegant approach leads us out of the safe haven into uncharted waters of experimental extensions.

The most concise implementation reuses the overlapping tricks that were discussed in Sec. 6, which makes the solution GHC-specific:

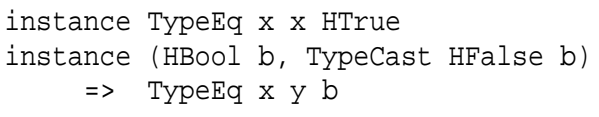

Here we take advantage of TypeCast, which we define next. 


\section{Reification of type unification}

The class TypeCast was introduced in Sec. 6 and further employed in Sec. 7. TypeCast $x$ y differs from just type equality TypeEq $x$ y HTrue as follows. If TypeCast $x$ y succeeds, then the two types are unified. The difference between unification and just equality emerges when the types are not grounded, i.e., when they contain uninstantiated type variables. The types [a] (e.g., of the polymorphic constant [] ) and [Bool] are unifiable, but they are not equal. TypeEq cannot establish equality for ungrounded types; however it can establish disequality in case the schemas are sufficiently instantiated to determine that they are not equal.

The most generic implementation of TypeCast, which works for both Hugs and GHC, is as follows:

instance TypeCast $\mathrm{x} \times$ where typeCast $=$ id

For this implementation to work, we need to import it at a higher level in the module hierarchy than all clients of the class TypeCast. Otherwise, type simplification will turn constraints of the form TypeCast $\mathrm{x} y$ into the form TypeCast $\mathrm{x} \mathrm{x}$, and thereby inline the unification. We refer to App. D, where we give another implementation of TypeCast, which does not require separate compilation. This time, we effectively delay the simplification step with the help of two auxiliary classes. It seems that this delay of type simplification is at the core of all attempts at type-safe cast or type equality (e.g., [4]).

A specific property of our TypeCast is that it allows us to control type improvement on a per-instance basis, as the polymorphism benchmark for TIPs showed in Sec. 7. So the utility of TypeCast goes strictly beyond a generic implementation of TypeEq.

\section{Related work}

\section{Heterogeneous lists}

Type-level list-processing is a relatively obvious opportunity once we get hold on faked dependently typed programming in Haskell, as pioneered by Hallgren and McBride [12, 21]. For instance, homogeneous type-level vectors are considered in [21]. The idea of heterogeneous type-level constructors (what we call HNil and HCons) occurs elsewhere in the literature. In App. $\mathrm{H}$ of [9], Duck et al. motivate their CHR-based model of functional dependencies by operating on such lists using numeral-based access (similar to our's in Sec. 4); Sulzmann also gives a related implementation in the Haskell-style language Chameleon [33]. In [22, 23], Neubauer et al. motivate Haskell extensions for a functional notation of functional dependencies, and for functional logic overloading. The authors consider examples like type-level functions append and length, as well as record-like operations. By contrast, our goal was to explore the various kinds of access operations for heterogeneous collections: list processing, numeral-based, label-based, and type-based operations. HLIST is the first heterogeneous collection library to the best of our knowledge.

\section{Type-indexed rows}

Shield and Meijer have studied the type theory of extensible records and variants starting from a more basic principle, namely typeindexed rows (TIRs) [31]. A TIR is nothing but a type expression that enumerates types. This resembles HLists, but TIRs do not comprise any values. So we could go for constructor-less datatypes:

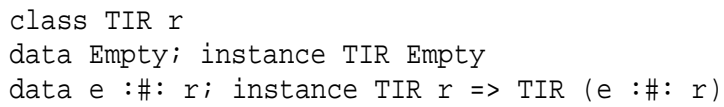

A TIR is well-formed if the enumerated types are distinct. Wellformedness corresponds to our HTypeIndexed constraint. Shield and Meijer provide type-level operators $A L L$ and $O N E$ that, given a TIR, derive types for type-indexed products (TIPs; recall Sec. 7) and type-indexed co-products (TICs; see App. C for the HLIST implementation of TICs). We could redefine our datatypes for TIPs and TICs such that they take a TIR as parameter, but these definitions and their usage would be more complicated in Haskell. Shield and Meijer argue that, conceptually, a newtype-like mechanism is sufficient for labelling. Our development provides labels as first-class citizens, and we can provide labelled collections without reference to general type-indexing (i.e., numeral indexing is sufficient). Our Haskell-based reconstruction of TIPs and TICs does not require new language extensions.

\section{Extensible records}

Foundations of extensible records have been studied intensively. Several Haskell language extensions have been proposed [10, 31, 29], alike for other languages, e.g., (S)ML $[6,30]$. There are also record calculi by Bracha, Ohori and others $[5,24]$. There are related type systems, e.g., for relational algebra [14]. We have shown that we can reconstruct extensible records in Haskell starting from simpler notions; in particular: heterogeneous lists and equality and of type-level naturals. We cover all typical record operations. We have also defined subtyping constraints in our framework.

Labels, values and records are all first-class citizens in HLIST. So we can write abstractions that take and produce entities of all these kinds. For instance, here is an operation to rename a record label:

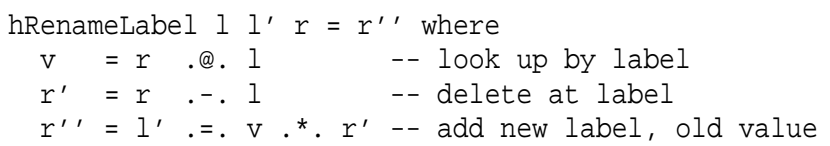

\section{Type equality and type cast}

In our development of heterogeneous collections, we rely on observability of type equality. Also, we employed a reified type unification ('type-level type cast) in a few places. Related expressiveness has been studied in the context of intensional polymorphism [13], dynamic typing [2, 3], and universal representations [36]. Some more recent Haskell-biased work on these notions $[34,8,4]$ is not directly usable for our purposes. These approaches either require the programmer to use type representations, or they make a closed-world assumption with regard to the covered types, or they are focused on sums-of-products (as opposed to the immediate coverage of Haskell's newtypes and datatypes), or they involve existential quantification (which makes it difficult to perform more arbitrary operations on elements in the collections). Most notably, we require a type cast that is resolved at typechecking time; run-time would be too late.

\section{Haskell's type classes}

Multi-parameter classes [7, 15, 16, 28] with functional dependencies $[17,9]$ are crucial for type-level programming in Haskell. These typing notions are reasonably understood. There is an ongoing debate if instance selection should be programmable by using constraint-handling rules or functional logic evaluation [32, 23]. Also, the mere notation for encoding type-level functions could perhaps be improved [22]. We have considered using overlapping instances for the definition of some access operations, but ultimately we eliminated use of this debated extension in a systematic manner.

\section{Statically enforced invariants}

The TIP newtype is an example of a data structure with a statically checked invariant (i.e., uniqueness). Okasaki and others have worked on statically assuring invariants of complex data types, e.g., 
that a matrix is square [25]. These examples normally rely on cleverly chosen data constructors, which make it impossible to construct "wrong" data structures. Our approach is different: type classes let us impose static constraints irrespective of data constructors. Indeed, we use the same data constructor HCons to build heterogeneous lists with and without duplicates. We express the constraints in types (sometimes, in phantom types). Our approach does not require extraordinary cleverness in the design of data representation. Furthermore, in the case of constraints encoded in phantom types, there is no run-time or -space overhead of storing and traversing chains of data constructors (TIP is just as efficient as HList). Because TIP is essentially HList, we were able to trivially lift all list-processing functions to TIPs. Statically checking complex invariants on data structures, such as well-formedness of red-black trees and size-boundaries of lists, is a known application of dependently typed programming [35]. The latter requires non-trivial extensions to a programming language. We have shown that certain invariants, e.g., size boundaries for HLists, or uniqueness in TIPs, can be statically expressed in Haskell's type system already.

\section{Conclusion}

We have systematically developed a Haskell library over stronglytyped data structures for heterogeneous collections - lists, arrays, extensible records, and others. The composition of such a data structure, e.g., the types of all elements, is manifest in its type. This makes it possible to strongly type the operations on collections, e.g., look-ups, updates, insertions, and projections. The name of the library, HLIST, emphasises that all data structures are built from typeful heterogeneous lists. We have defined restricted collections, e.g., TIPs, constrained by the requirement that no two elements may have the same type. The constraints are again manifest in the type of the collections and are enforced by the type checker.

The immediate application of our HLIST library is a database access library that covers SQL92, returns the query results as a stream of records, and statically checks that all the queries are consistent with the database schema.

The implications of the library HLIST turn out far reaching, and are still under active investigation. Our TIPs and records are extensible and offer subtyping polymorphism. Our records have first-class labels that can be reused across several record types. We notice that HLIST is implemented in Haskell with only common extensions. Hence the HLIST library addresses the challenge for better Haskell records, without breaking existing programs, as articulated by Simon Peyton Jones at the Haskell Workshop 2003 [11]. Our records also let us implement has/lacks, record concatenation, length vs. depth subtyping. We can now experiment with these features in real programs - again, without requiring any language extension.

Extensible TIPs and records can be the foundation of the genuine object system. The latter offers subtyping polymorphism (cf. OCaml) as opposed to the class-bounded polymorphism of Haskell. It is remarkable that type classes themselves were instrumental in implementing open TIPs. Extensible records can also be elaborated to provide strongly typed keyword arguments with reusable labels. That is, function arguments can be addressed by keywords, and these arguments can be optional or mandatory. The HLIST source distribution demonstrates keyword arguments. Dual to TIPs are open TICs, offering us dynamics with a statically-checkable constraint on the sort of types encapsulated in the dynamic envelope (cf. App. C). The lists, TIPs, TICs and records of the HLIST library can also be employed in typeful foreign-function interfaces and in XML processing.

Our code relies on the most common Haskell extensions; the use of overlapping instances can be circumvented. In fact, a generic im- plementation of the predicate TypeEq for type equality would still rely on overlapping in a single location. We can also implement TypeEq in a portable but non-generic manner relying on one instance per user-defined datatype. Our development suggests that a fundamental solution could be to offer type equality as a primitive in Haskell. We have also identified the utility of reified type unification (or 'type-level type cast') as a tool for type improvement more fine-grained than functional dependencies. More research is needed to deliver foundational clarifications.

\section{Acknowledgements}

We thank Chung-chieh Shan, Martin Sulzmann and the PC of the Haskell Workshop 2004 for very helpful comments and feedback.

\section{References}

[1] This paper's web site http://www.cwi.nl/ ralf/HList/, 2004. This site provides an extended paper version with extra appendicies that could not be included into the Haskell workshop paper. This site also provides a source code distribution for the GHC and Hugs implementations of Haskell.

[2] M. Abadi, L. Cardelli, B. Pierce, and G. Plotkin. Dynamic typing in a statically-typed language. In 16th ACM Conference on Principles of Programming Languages, pages 213227, Jan. 1989.

[3] M. Abadi, L. Cardelli, B. Pierce, and D. Remy. Dynamic typing in polymorphic languages. In Proceedings of the 1992 ACM Workshop on ML and its Applications, pages 92-103, San Francisco, June 1992.

[4] A. Baars and S. Swierstra. Typing dynamic typing. In Proceedings of the seventh ACM SIGPLAN International Conference on Functional Programming, pages 157-166. ACM Press, 2002.

[5] G. Bracha and G. Lindstrom. Modularity Meets Inheritance. In Proceedings: 4th International Conference on Computer Languages, pages 282-290. IEEE Computer Society Press, 1992.

[6] F. Burton. Type extension through polymorphism. TOPLAS, 12(1):135-138, 1990.

[7] K. Chen, P. Hudak, and M. Odersky. Parametric type classes. In Proceedings of the 1992 ACM Conference on LISP and Functional Programming, pages 170-181. ACM Press, 1992.

[8] J. Cheney and R. Hinze. A lightweight implementation of generics and dynamics. In Proceedings of the ACM SIGPLAN Workshop on Haskell, pages 90-104. ACM Press, 2002.

[9] G. Duck, S. Peyton Jones, P. Stuckey, and M. Sulzmann. Sound and Decidable Type Inference for Functional Dependencies. In D. Schmidt, editor, Proceedings, 13th European Symposium on Programming, ESOP 2004, Barcelona, Spain, March 29 - April 2, 2004, volume 2986 of LNCS, pages 4963. Springer-Verlag, 2004.

[10] B. Gaster and M. Jones. A Polymorphic Type System for Extensible Records and Variants. Technical report NOTTCSTR-96-3, University of Nottingham, Department of Computer Science, Nov. 1996.

[11] H. Nilsson. The Future of Haskell discussion at the Haskell Workshop, 2003. http://www.mail-archive. com/haskell @haskell.org/msg13366.html.

[12] T. Hallgren. Fun with functional dependencies. In Joint Winter Meeting of the Departments of Science and Computer Engineering, Chalmers University of Technology and Goteborg University, Varberg, Sweden, Jan. 2001, 2001. http:

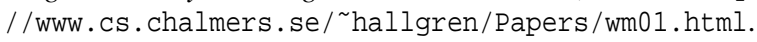


[13] R. Harper and G. Morrisett. Compiling polymorphism using intensional type analysis. In 22nd ACM Symposium on Principles of Programming Languages (POPL'95), pages 130-141. ACM Press, Jan. 1995.

[14] E. W. J. Van den Bussche. Polymorphic type inference for the relational algebra. Journal of Computer and System Sciences, 64:694-718, 2002. An extended abstract appeared in PODS' 99 .

[15] M. Jones. A theory of qualified types. In Symposium proceedings on 4th European symposium on programming, pages 287-306. Springer-Verlag, 1992.

[16] M. Jones. Simplifying and improving qualified types. In Proceedings of the seventh international conference on Functional Programming Languages and Computer Architecture, pages 160-169. ACM Press, 1995.

[17] M. Jones. Type classes with functional dependencies. In Proceedings of the 9th European Symposium on Programming Languages and Systems, pages 230-244. Springer-Verlag, 2000.

[18] O. Kiselyov. Polymorphic stanamically balanced binary trees, 2003. http://www.haskell.org/pipermail/haskell/ 2003-April/011621.html.

[19] R. Lämmel and S. Peyton Jones. Scrap your boilerplate: a practical design pattern for generic programming. ACM SIGPLAN Notices, 38(3):26-37, Mar. 2003. Proc. of the ACM SIGPLAN Workshop TLDI 2003.

[20] D. Leijen and E. Meijer. Domain specific embedded compilers. In Proceedings of the 2 nd conference on Domain-Specific Languages, pages 109-122. ACM Press, 1999.

[21] C. McBride. Faking It (Simulating Dependent Types in Haskell), July 2002.

[22] M. Neubauer, P. Thiemann, M. Gasbichler, and M. Sperber. A Functional Notation for Functional Dependencies. In Proc. 2001 ACM SIGPLAN Haskell Workshop, Firenze, Italy, September 2001, pages 101-120, 2001.

[23] M. Neubauer, P. Thiemann, M. Gasbichler, and M. Sperber. Functional logic overloading. In Proceedings of the 29th ACM SIGPLAN-SIGACT symposium on Principles of Programming languages, pages 233-244. ACM Press, 2002.

[24] A. Ohori. A polymorphic record calculus and its compilation. ACM TOPLAS, 17(6):844-895, 1995.

[25] C. Okasaki. From fast exponentiation to square matrices: an adventure in types. In Proceedings of the fourth ACM SIGPLAN International Conference on Functional Programming, pages 28-35. ACM Press, 1999.

[26] C. Okasaki. An Overview of Edison. In G. Hutton, editor, Electronic Notes in Theoretical Computer Science, volume 41. Elsevier, 2001.

[27] S. Peyton Jones. Adding Ord constraint to instance Monad Set?, 2004. http://www. haskell.org/pipermail/ haskell-cafe/2004-March/005998.html.

[28] S. Peyton Jones, M. Jones, and E. Meijer. Type classes: exploring the design space. In J. Launchbury, editor, Haskell workshop, Amsterdam, 1997.

[29] S. Peyton Jones and G. Morrisett. A proposal for records in Haskell, 24 Feb. 2003. Online document: http://research.microsoft.com/ simonpj/Haskell/ records.html.

[30] D. Remy. Type inference for records in natural extension of $\mathrm{ml}$. In Theoretical aspects of object-oriented programming: types, semantics, and language design, pages 67-95. MIT
Press, 1994.

[31] M. Shields and E. Meijer. Type-indexed rows. In Proceedings of the 28th ACM SIGPLAN-SIGACT symposium on Principles of Programming Languages, pages 261-275. ACM Press, 2001.

[32] P. Stuckey and M. Sulzmann. A theory of overloading. In Proceedings of the seventh ACM SIGPLAN International Conference on Functional Programming, pages 167-178. ACM Press, 2002.

[33] M. Sulzmann et al. Chameleon, 2004. Web site http://www. comp.nus.edu.sg/ ^sulzmann/chameleon/.

[34] S. Weirich. Type-safe cast: (functional pearl). In Proceedings of the fifth ACM SIGPLAN International Conference on Functional Programming, pages 58-67. ACM Press, 2000.

[35] H. Xi and F. Pfenning. Dependent types in practical programming. In Proceedings of the 26th ACM SIGPLAN-SIGACT symposium on Principles of Programming Languages, pages 214-227. ACM Press, 1999.

[36] Z. Yang. Encoding types in ML-Like languages. In M. Berman and S. Berman, editors, Proceedings of the third ACM SIGPLAN International Conference on Functional Programming (ICFP'98), volume 34, 1 of ACM SIGPLAN Notices, pages 289-300, New York, Sept. 27-29 1998. ACM Press.

\section{A Some trivial list-processing operators}

We will now transpose several normal list-processing operators to the heterogeneous situation.

\section{Transposition of head and tail}

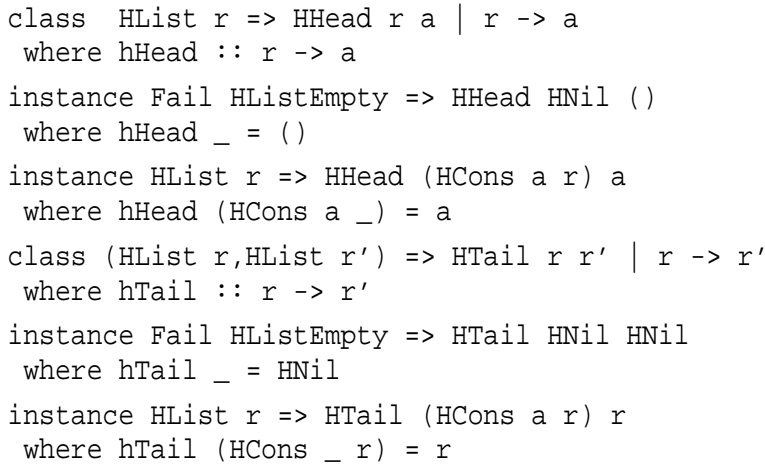

In the above instances, we use the same technique for error messaging as explained in Sec. 6. That is, we employ the Fail class to handle invalid applications of the operations. In particular, there is an error message HListEmpty, whenever we attempt to access an empty list where a nonempty list is needed. Thus, we have: $\begin{array}{ll}\text { class Fail x } & -- \text { no instances! } \\ \text { data HListEmpty } & -- \text { no structure! }\end{array}$

\section{Transposition of null}

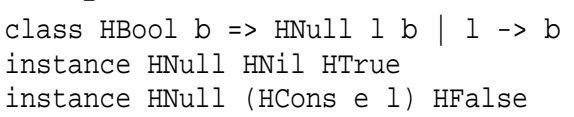

\section{Transposition of length}

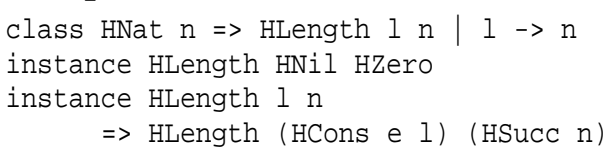




\section{B A heterogeneously typed fold operator}

We go for the fold operation because it is the ultimate example of a higher-order list-processing function. We dedicate a class HFoldr to right-associative folding. The HFoldr instances will lift the defining equations for foldr to the class level:

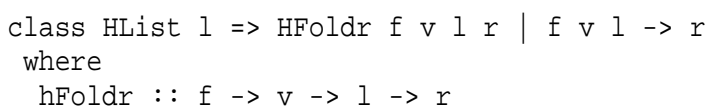

The instance for empty lists is trivial:

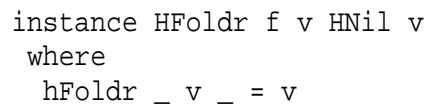

The instance for HCons follows the normal foldr again, but we assume that function application is modelled by an extra class HApply. This allows us to use hFoldr for functions that require specific constraints on the involved types:

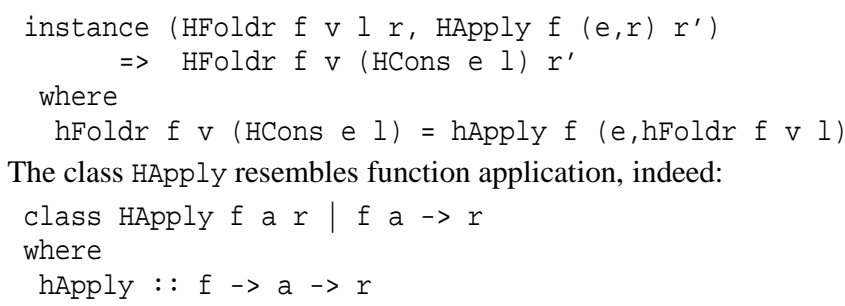

For instance, we can now redefine hAppend in terms of hFoldr:

hAppend $11^{\prime}=$ hFoldr ApplyHCons $l^{\prime} l$

The datatype ApplyHCons stands for "application of HCons":

data ApplyHCons =

ApplyHCons -- a proxy for instance selection

This meaning of ApplyHCons is registered as an HApply instance:

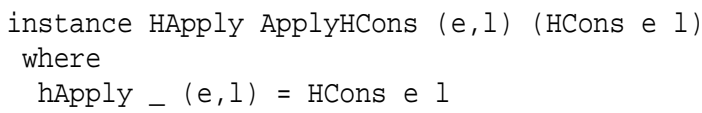

\section{Type-indexed co-products}

We will now dualise TIPs to arrive at so-called type-indexed sums (or co-products; TICs). A TIC-typed data structure holds a datum of one out of a fixed collection of types. So at the value level, a TICtyped data structure is not really a collection, but just one datum. However, at the type level we use a list of type proxies to maintain the valid element types of a specific TIC type, and thereby we can restrict construction and destruction of TIC-like data structures.

\section{A TIC demo}

We first define an actual TIC type, namely one that models various element types for collections related to the animals in the 'foot-nmouth' database:

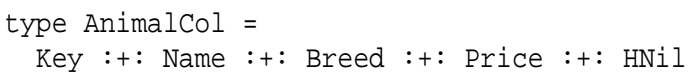

Here we use ": + :" rather than " : *:" to point out that we are interested in a type-indexed co-product rather than a product. We can now construct actual TIC-like data. For instance:

ghci-or-hugs $>$ let myCol = mkTIC Cow : : TIC AnimalCol We can also destruct myCol. If we ask for the 'right' type, then destruction succeeds with a result of the form Just . ...; otherwise we obtain Nothing:

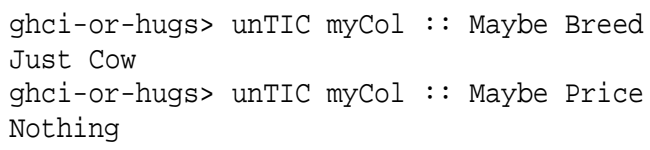

Most notably, TICs restrict destruction with regard to static typing: ghci-or-hugs> unTIC myCol : : Maybe String Type error ...

\section{Sequences of type proxies}

We used the alias ": + :" above to enumerate the summands of a TIC type. In fact, ": $+:$ " is constructed such that it lines up proxy types in a sequence. Value types would be misleading and confusing here because the sequence of summands is meant for nothing but listing 'options'. So the alias is defined as follows:

type e :t: 1 = HCons (Proxy e) 1

The actual property of a type sequences to consist only of proxy types is easily specified.

class HTypeProxied 1

instance HTypeProxied HNil

instance HTypeProxied 1

$$
\Rightarrow \text { HTypeProxied (HCons (Proxy e) 1) }
$$

\section{TICs as constrained dynamics}

The demo suggests that a TIC is more constrained than the type Dynamic. So in turn, one can define more constrained collection types than just [Dynamic] or String $\rightarrow$ Dynamic. There exist different implementations of TICs, but we will favour here one that indeed directly employs Haskell's dynamics at the value level.

A TIC type is then of the following form:

$$
\text { data TIC } 1 \text { = TIC Dynamic -- to be constrained }
$$

The phantom type parameter 1 of TIC enumerates the admitted types that can be injected into this TIC, and that can be subject to extraction attempts. The public constructor for TICs (aka injection) lists all the necessary constraints:

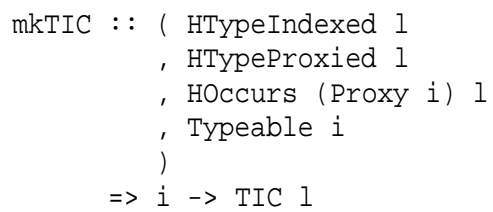

The HTypeIndexed and HTypeProxied constraints require that $l$ is a type-indexed sequences of type proxies. The HOccurs constraint ensures that the proxy type of the injected value $i$ is covered by the sequence of proxies 1 . Finally, the Typeable constraint allows us to use Haskell's module Data. Dynamic.

It remains to define destruction (or projection), which happens to simply invert the constrained value-to-dynamics conversion:

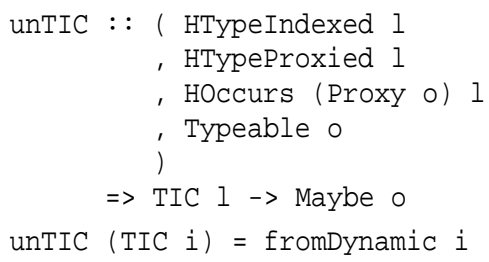




\section{Generic type unifi cation cont'd}

The class TypeCast was described in the subsection 'Reification of type unification' of Sec. 9.

$$
\begin{aligned}
& \text { class TypeCast } a b \mid a->b, b->a \\
& \text { where typeCast : : } a->b
\end{aligned}
$$

That section showed the most straightforward implementation of that class: a single instance TypeCast $\mathrm{x} \times$ with the method typeCast being just the identity. However, that simple implementation was difficult to use. Separate compilation had to be put to use in some tricky way. Indeed, recall the following example of using TypeCast from Sec. 7:

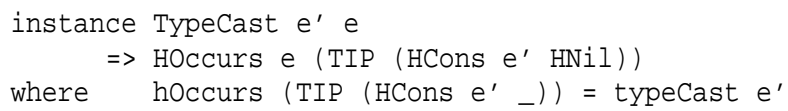

When the compiler sees the instance TypeCast $\mathrm{x} \times$ and combines that with the functional dependencies $a->b, b->a$ of the class, the compiler infers that the two parameters of Typecast must be the same. That conclusion is correct - the type cast is meant to be an isomorphism on types (in fact, the identity function). What is troublesome is that the type checker applies that conclusion - as a type simplification rule - to the HOccurs instance above and infers that e must be $e^{\prime}$. That is a problem however: if a type signature contains distinct type variables, one should be able to instantiate them, at least in principle, with distinct types. Otherwise, the inferred type is less polymorphic than the explicit signature prescribes.

This is the same sort of error that arises in the following code:

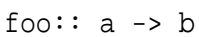

When processing the instance declaration HOccurs, the compiler eagerly applies the correct type simplification rule - the two parameters of TypeCast must be the same - and infers that two type variables $e$ and $e^{\prime}$ must be the same. The eagerness creates the problem. We would like to delay the type simplification until after the instance HOccurs has been selected and e and $e^{\prime}$ have been instantiated. In other words, we would like to unify the types that $\mathrm{e}$ and $\mathrm{e}^{\prime}$ are instantiated with, rather than the two type variables themselves.

To keep the compiler from applying the type simplification rule too early, we should prevent the early inference of the rule from the instance of TypeCast in the first place. For example, we may keep the compiler from seeing the instance TypeCast $\mathrm{x} x$ until the very end. That is, we place that instance in a separate module and import it at a higher level in the module hierarchy than all clients of the class TypeCast. That was the approach described in Sec. 9.

We will now give another implementation of TypeCast, which does not require separate compilation. It effectively delays the simplification step with the help of two auxiliary classes.

Our new implementation must keep the semantics of the constraint: TypeCast a b should hold if and only if the type corresponding to a can be unified with the type corresponding to $b$. On the other hand, we need to allow for polymorphism and pretend that in a constraint Typecast $\mathrm{a}, \mathrm{b}, \mathrm{b}$ may be something other than $\mathrm{a}-$ so to keep the typechecker from unifying the type variables $a$ in $b$ in occurrences of that constraint. Fortunately, the type system is not very smart: when choosing the instances the type-checker looks only at the syntactic form of the type terms involved. Therefore, to fool the type-checker into thinking that TypeCast $a \mathrm{~b}$ is more polymorphic than it really is, we introduce a series of redirections and eventually arrive at the following implementation.

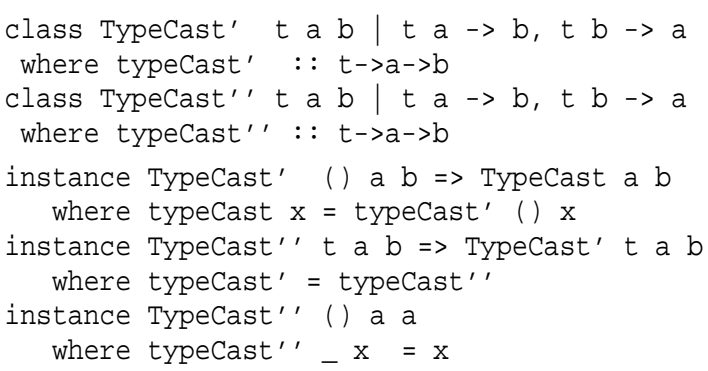

The auxiliary classes TypeCast' and TypeCast' ' have an extra, dummy type parameter, which we instantiate to () in the instances. Any other ground type would have sufficed. The key to solving the polymorphism quandary is the last instance TypeCast' ' () a a. It signifies that in the constraint TypeCast' ' $t a b, b$ is not necessarily a, because $t$ can be something other than (). Semantically, though, it can never be anything but. However, the type-checker cannot see that and remains satisfied.

Alas, this implementation is specific to GHC; it does not work in Hugs because of the peculiarities of that system with regard to multi-parameter type classes and functional dependencies, which we briefly hinted at in Sec. 6. That shows that multi-parameter type classes with functional dependencies are hard to get right.

While this code works in GHC and is logically sound, we have to admit that we turned the drawbacks of the type-checker to our advantage. This leaves a sour after-taste. We would have preferred to rely on a sound semantic theory of overloading rather than on playing games with the type-checker. Hopefully, the results of the foundational work by Sulzmann and others [32, 23] will eventually be implemented in all Haskell compilers. 